\title{
Macrophage proliferation distinguishes 2 subgroups of knee osteoarthritis patients
}

\author{
Matthew J. Wood, ${ }^{1,2}$ Adam Leckenby, ${ }^{1,2}$ Gary Reynolds, ${ }^{1,2,3}$ Rachel Spiering, ${ }^{1,2}$ Arthur G. Pratt, ${ }^{1,2,3}$ \\ Kenneth S. Rankin,, ${ }^{1,3}$ John D. Isaacs,, ${ }^{1,2,3}$ Muzlifah A. Haniffa, ${ }^{1,3,4}$ Simon Milling, ${ }^{2,5}$ \\ and Catharien M.U. Hilkens $\mathbf{s}^{1,2,3}$ \\ IInstitute of Cellular Medicine, Newcastle University, United Kingdom. ${ }^{2}$ Arthritis Research UK Rheumatoid Arthritis \\ Pathogenesis Centre of Excellence, Glasgow, Birmingham, Newcastle, United Kingdom. ${ }^{3}$ NIHR Newcastle Biomedical \\ Research Centre and ${ }^{4}$ Department of Dermatology, Newcastle Hospitals NHS Foundation Trust and Newcastle \\ University, Newcastle upon Tyne, United Kingdom. Institute of Infection, Immunity and Inflammation, Glasgow \\ University, United Kingdom.
}

Osteoarthritis (OA) is a leading cause of disability, globally. Despite an emerging role for synovial inflammation in $\mathrm{OA}$ pathogenesis, attempts to target inflammation therapeutically have had limited success. A better understanding of the cellular and molecular processes occurring in the OA synovium is needed to develop novel therapeutics. We investigated macrophage phenotype and gene expression in synovial tissue of $\mathrm{OA}$ and inflammatory-arthritis (IA) patients. Compared with IA, OA synovial tissue contained higher but variable proportions of macrophages $(P<0.001)$. These macrophages exhibited an activated phenotype, expressing folate receptor- 2 and CD86, and displayed high phagocytic capacity. RNA sequencing of synovial macrophages revealed $20 \mathrm{~A}$ subgroups. Inflammatory-like OA (iOA) macrophages are closely aligned to IA macrophages and are characterized by a cell proliferation signature. In contrast, classical OA (COA) macrophages display cartilage remodeling features. Supporting these findings, when compared with cOA, IOA synovial tissue contained higher proportions of macrophages $(P<0.01)$, expressing higher levels of the proliferation marker Ki67 $(P<0.01)$. These data provide new insight into the heterogeneity of $O A$ synovial tissue and suggest distinct roles of macrophages in pathogenesis. Our findings could lead to the stratification of $\mathrm{OA}$ patients for suitable disease-modifying treatments and the identification of novel therapeutic targets.

Conflict of interest: The authors have declared that no conflict of interest exists.

License: This work is licensed unde the Creative Commons Attribution 4.0 International License. To view a copy of this license, visit http:// creativecommons.org/licenses/ by/4.0/

Submitted: October 3, 2018 Accepted: December 18, 2018 Published: January 24, 2019

\section{Reference information:} JCI Insight. 2019;4(2):e125325. https://doi.org/10.1172/jici. insight.125325.

\section{Introduction}

Osteoarthritis (OA) is the most prevalent form of arthritis and a leading cause of disability in the Western world. Its incidence has doubled since the mid-20th century $(1,2)$. OA is characterized by the progressive destruction of cartilage, causing pain and disability. Attempts to develop disease-modifying drugs have so far failed, leaving joint replacement as the final therapeutic option (3). In 2015, >90,000 patients received a total knee replacement in the United Kingdom; this figure is expected to double over the next decade $(4,5)$. Although outcomes of total joint replacement are positive for the majority, up to $20 \%$ are dissatisfied due to persistent pain or stiffness, which may be related to inflammation in the residual soft tissues $(6,7)$. There is, therefore, a requirement to improve our understanding of OA pathogenesis in order to develop novel interventions.

The pathogenesis of primary OA remains poorly understood, but it has become clear that inflammatory processes are involved. Synovial inflammation present in OA patients is usually low grade and does not lead to overt systemic inflammation (8). However, there is a clear positive correlation between synovial inflammation and clinical features of OA, such as pain, swelling, and cartilage damage (9-15). Recently, Soul et al. (16) suggested that OA patients are heterogeneous and could be stratified into 2 major patient subgroups based on the gene expression of knee articular cartilage, showing altered patterns of matrix protein gene expression (16). Indeed, clinical heterogeneity in OA pathogenesis is increasingly becoming recognized (1720). However, OA patients are not currently stratified for clinical trials based on synovitis or inflammatory biomarkers (21) because the mechanisms governing synovial inflammation in OA are not sufficiently understood. Clinical trials with antiinflammatory treatments in OA have so far been disappointing, which may 
reflect underlying OA disease heterogeneity $(21,22)$. Stratification of OA patients based on inflammatory processes in the synovium may be helpful for matching the most appropriate antiinflammatory treatments to the individual OA patient.

Several lines of evidence implicate macrophages in OA pathogenesis. Within the normal synovium, macrophages are the main immune cell type (23) and are most likely to be the front-line cells that sense joint damage. In response to damage-associated molecular patterns (DAMP) signals, macrophages in the OA synovium are reported to contribute to cartilage destruction and osteophyte formation by producing promatrix metalloproteinases (MMPs), and cytokines including IL-1 $\beta$, TNF- $\alpha$, and TGF- $\beta$ (24-26). Histological studies have observed enhanced numbers of macrophages in OA synovium (26-28), which correlated with the degree of synovial angiogenesis (26), a hallmark of synovitis. Recently, a noninvasive imaging study with a folate receptor- $\beta$-based agent has shown that the quantity of activated macrophages in the knee of OA patients correlated with disease severity and progression (29), implicating these cells in OA pathogenesis. Depletion of macrophages in animal models of OA can markedly reduce osteophyte formation $(24,25)$ but can also enhance synovial inflammation (30), suggesting diverse roles of macrophages in OA pathogenesis. Indeed, macrophages are known to exert both proinflammatory and antiinflammatory/ tissue repair functions (31). Recent studies have revolutionized our understanding of tissue macrophage heterogeneity, revealing how their diverse functions are modulated by the local tissue environment (31-33).

In order to design new therapeutic approaches that selectively target the pathogenic function of synovial macrophages, we first need to understand which of their functions contribute to OA pathogenesis and how they interact with their environment. To address this aim, we have profiled gene expression by synovial macrophages from OA and IA patients. These data reveal 2 distinct subgroups of OA patients, one of which is characterized by macrophage proliferation.

\section{Results}

Identification and enumeration of synovial tissue cell populations. We identified synovial macrophages and other immune cell subsets by flow cytometry (Figure 1A). Autofluorescence has previously been used as a marker of tissue macrophages in skin $(34,35)$. Within the autofluorescent fraction, a putative HLA-DR ${ }^{+} \mathrm{CD} 14^{+}$ synovial macrophage population was identified (T1). These expressed other markers of macrophages (e.g., CD64 and CD11c) and morphological features including large size and abundant cytoplasm with multiple vacuoles (Figure 1B). In contrast, within the nonautofluorescent fraction, a population of HLA-DR ${ }^{+}$cells were likely to represent tissue monocytes (T3) and DCs (T4), as indicated by surface marker expression (CD11c, CD14 for monocytes; CD11c, CD1c for DCs) as well as their morphological appearance (Figure 1, $\mathrm{A}$ and $\mathrm{B}) . \mathrm{CD}^{+} \mathrm{T}$ cells were identified within the $\mathrm{CD} 3^{+}$fraction (T2). Full surface marker phenotypes of synovial cell subsets are listed in Table 1 .

Manual and computational approaches were employed to quantify the cellular distribution in the synovial tissue of IA and OA patients (Figure 1, C-E). viSNE plots, based on the t-distributed stochastic neighbor embedding (tSNE) algorithm, were used to visualize representative cell contributions and relationships from high-dimensional flow cytometry data. Cell clusters were determined by protein expression of CD45, HLA-DR, CD3, CD16, CD11c, CD14, CD4, and CD1c, as well as autofluorescence. Activated $\mathrm{T}$ cells were identified by expression of HLA-DR. When comparing the cellular distribution in the synovial tissue of our IA and OA patient cohorts (OA, $n=64$; IA, $n=19$; Supplemental Table 1; supplemental material available online with this article; https://doi.org/10.1172/jci.insight.125325DS1), OA consisted of fewer $\mathrm{CD}_{4} 5^{+}$cells (Supplemental Figure 1), with macrophages representing the main immune cell subset (Figure 1, C-E). However, this proportion of macrophages was highly variable between OA patients (Figure 1E). IA synovial tissue contained larger proportions of $\mathrm{CD}^{+} \mathrm{T}$ cells, represented in blue by viSNE analyses (Figure 1D) and by manual quantification (Figure 1E). Furthermore, a proportion of IA $\mathrm{CD}^{+} \mathrm{T}$ cells had an activated phenotype (Figure $1 \mathrm{D}$ ), while activated $\mathrm{CD} 4^{+} \mathrm{T}$ cells could not be detected in OA synovial tissue. A significant difference was measured in BMI between OA and IA patient cohorts. To address whether BMI was inducing the infiltration of macrophages into the synovium of OA patients, we stratified the proportion of macrophages, in addition to monocytes, DCs, and T cells identified by flow cytometry, with BMI. No significant differences were measured (data not shown).

Phenotype and functional assessment of synovial macrophages. We next determined functional attributes and phenotype of synovial macrophages. The ability of macrophages to phagocytose fluorescent latex beads was measured by flow cytometry. Synovial macrophages displayed comparable phagocytic capacity to in 
Table 1. Full surface marker phenotype of cell subsets isolated from synovial tissue and peripheral blood

\begin{tabular}{|c|c|}
\hline Cell population & Surface marker phenotype \\
\hline Macrophage (HLA-DR $\left.{ }^{+} \mathrm{CD}_{14}^{+}\right)$ & Live, $\mathrm{CD}^{-} 9^{-}, \mathrm{CD}^{2} 0^{-}, \mathrm{CD}^{\circ} 5^{+}, \mathrm{AF}^{+}, \mathrm{CD}^{+} 4^{+}, \mathrm{HLA}^{-\mathrm{DR}^{+}}$ \\
\hline T cell $\left(\mathrm{CD}^{+}\right)$ & Live, $\mathrm{CD}^{-} 9^{-}, \mathrm{CD}^{-} 0^{-}, \mathrm{CD}_{4} 5^{+}, \mathrm{AF}^{-}, \mathrm{HLA}^{-\mathrm{DR}^{-}, \mathrm{CD}^{+}, \mathrm{CD}^{-}, \mathrm{CD}^{+}}$ \\
\hline Monocyte (CD14+) & 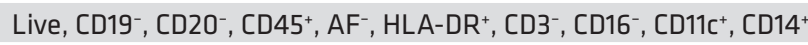 \\
\hline $\mathrm{DC}\left(\mathrm{CD}^{+} \mathrm{c}^{+}\right)$ & 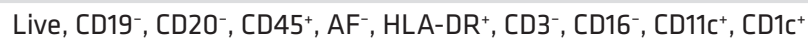 \\
\hline
\end{tabular}

vitro blood $\mathrm{CD} 14^{+}$monocyte-derived macrophages, indicating that the tissue-extracted cells retained this function (Figure 2A). Moreover, synovial macrophages were more efficient at phagocytosis than in vitro $\mathrm{CD}_{14}{ }^{+}$monocyte-derived DCs (Figure 2A) and synovial DCs (Figure 2B). To ensure that the beads were internalized, confocal microscopy was performed. Z-stack images were produced to allow visualization of the beads on the same field as the cell nucleus, in addition to high-contrast differential interference contrast microscopy (Figure 2, C and D). In addition to common macrophage markers HLA-DR, CD14, CD11c, and CD64, we found that synovial macrophages expressed CD86 and FOLR2 (Figure 2E). No significant difference was observed between OA and IA macrophages in the expression of the cell surface markers measured (Figure 2E) or in cell size (data not shown).

$R N A$ sequencing of synovial tissue macrophages identifies 2 distinct $O A$ subgroups. In order to identify any differences between synovial macrophages from OA and IA, we performed RNA sequencing (RNA-Seq) on synovial macrophages isolated from OA and IA patients (OA, $n=9$; IA, $n=3$; Supplemental Table 2). To verify the purity of macrophage samples sequenced, the expression of a set of macrophage genes and genes of potential contaminating cell subsets were measured (Figure 3A). All samples lacked expression of genes associated with other immune cell types, including B cells (B cell receptor; BCR, CD1c), DCs (CD1c), NK cells (nuclear adhesion molecule; NCAM1), stromal cells (THY1), and T cells ( $\mathrm{T}$ cell receptor $\alpha$ constant; TRAC). Additional DC gene sets were measured to further confirm purity of our HLA-DR ${ }^{+} \mathrm{CD}_{14}{ }^{+}$synovial macrophage population (Supplemental Figure 2). In contrast, all macrophage samples expressed genes associated with macrophages, including CD14, CD68, CSF1R, HLA-DRA, and MARCO. We evaluated the expression of genes characteristic of M1/M2 macrophages. However, we did not observe any clear correlation with M1/M2 macrophage genes in our dataset (Supplemental Figure 3). When plotting the principal components of these data, some clustering of IA samples was evident, but OA samples appeared to be highly diverse (Figure 3B). When plotting the top 500 most variable genes in a hierarchical heatmap, 2 distinct clusters are present: a group of OA samples and a group of OA and IA samples (Figure 3C). This differential clustering was still evident in the absence of the IA samples and remained after considering only the top 25 most variable genes (Supplemental Figure 4, A and B). In relation to the IA group, the distinct OA group was termed classical OA (cOA), whereas the OA subgroup that proved more similar to IA was designated inflammatory-like OA (iOA). Interestingly, these cOA and iOA groups correlate with the flow cytometry synovial tissue immune profiling data, giving further credence to our findings (Supplemental Figure 4C).

Gene expression pathway analysis suggests distinct disease mechanisms. Gene expression analysis revealed that 155 genes were differentially expressed between the cOA and iOA subgroups $(>1.5$ fold change; $P<0.05$, Benjamini-Hochberg multiple test corrected) (Figure 3D). Differential gene expression analysis between cOA vs. iOA and cOA vs. IA yielded the greatest number of differentially expressed genes, compared with OA vs. IA and iOA vs. IA, further supporting the gene expression similarities observed between iOA and IA (Supplemental Figure 5 and Supplemental Tables 3-6). Gene set enrichment analysis (GSEA) of the differentially expressed gene set from cOA vs. IA showed numerous genes involved in cartilage development and extracellular organization (Supplemental Table 5). Particularly, SMAD3 is common to many of these functional pathways. Indeed, $S M A D 3$ has been previously identified in genome-wide association studies (GWAS) and is associated with OA $(36,37)$. In contrast, when investigating genes overexpressed in iOA as compared with cOA, a strong cell cycle signature was identified (Supplemental Table 6). In line with the cell cycle signature of the iOA group, synovial tissue of this group contained a significantly increased proportion of synovial macrophages as compared with cOA synovial tissue (Figure 4B). After intersecting the differentially expressed gene sets across each comparison, the majority of upregulated genes were determined to be unique to each group (Figure 4A). However, nearly half of the downregulated genes 
A
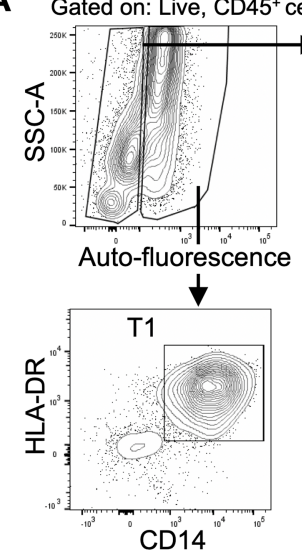

C

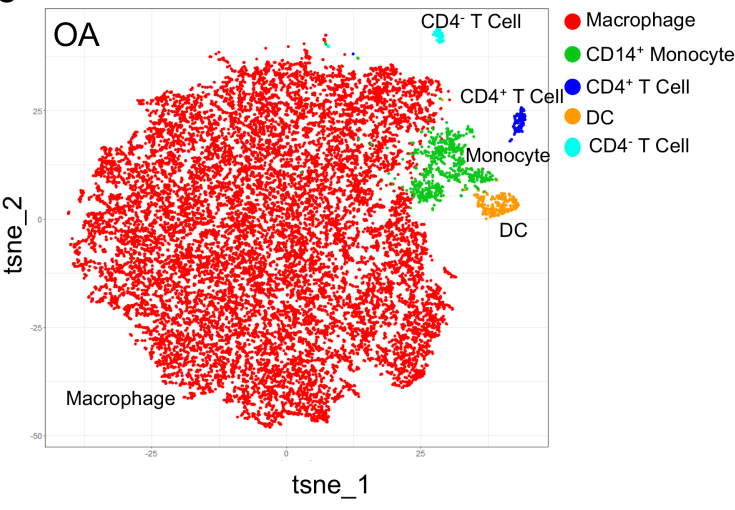

B
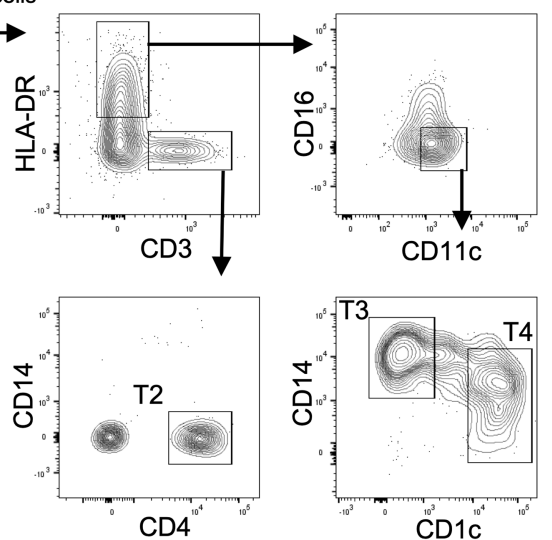

T1

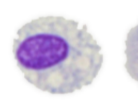

T2

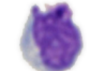

T3

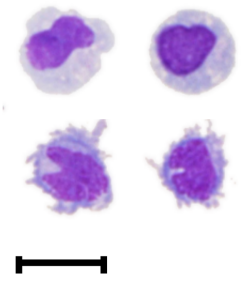

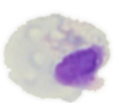

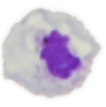

D

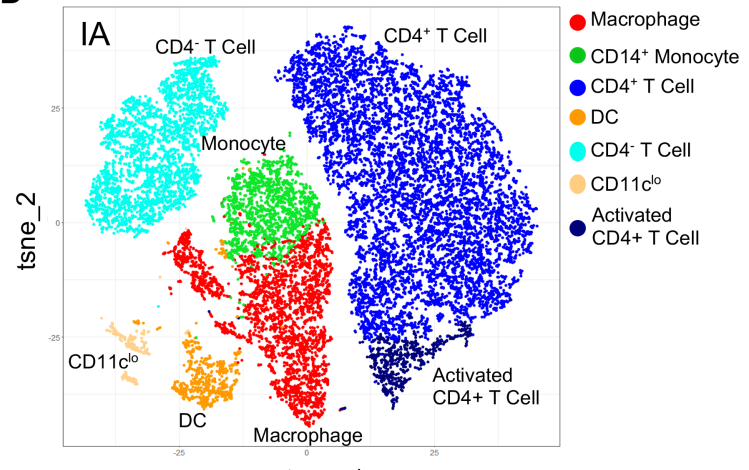

tsne_1
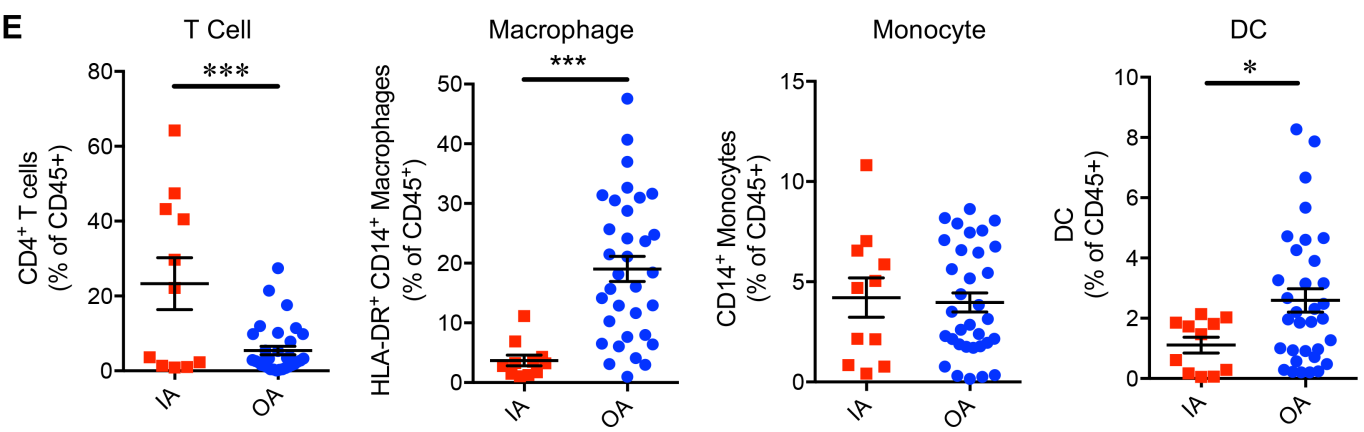

Figure 1. Characterization of synovial tissue cellular content in arthritis patients. Synovial tissue from OA total knee replacement and IA ultrasound guided biopsy was digested using optimized protocol. (A) Flow cytometry identification of synovial tissue HLA-DR ${ }^{+}$CD14 ${ }^{+}$macrophages (T1), CD4 ${ }^{+}$T cells (T2), CD14+ monocytes (T3), and DCs (T4). (B) Cytospin morphological assessment of synovial tissue cell subsets. Scale bar: $20 \mu \mathrm{m}$. Images are representative of 5 individual experiments. (C) tSNE analysis of flow cytometry data for OA synovial tissue mononuclear cells. Cell clusters were determined by both featurePlots and conventional flow cytometry gating on tSNE-analyzed FCS files; macrophage, T cell, monocyte, and DC clusters were determined, as indicated in Table 1. (D) tSNE analysis of flow cytometry data for IA synovial tissue mononuclear cells. Cell clusters were determined by both featurePlots and conventional flow cytometry gating on TSNE-analyzed FCS files; macrophage, T cell, monocyte, and DC clusters were determined as indicated in Table 1; CD11 ${ }^{10}$ cluster was determined by low expression of CD11c on HLA-DR+ population; activated CD4+ $\mathrm{T}$ cell cluster was determined by expression of HLA-DR by $C D 4^{+}$T cells. (E) Enumeration of $\mathrm{OA}$ and IA synovial tissue cell subsets as a percentage of total CD45+ cells. T cell, $n=32 ;$ macrophage, $n=45 ;$ monocyte, $n=45 ; \mathrm{DC}, n=45 .{ }^{*} P \leq 0.05,{ }^{* * *} P \leq 0.001$ by 2 -tailed unpaired $t$ test.

were shared between the cOA vs. IA and cOA vs. iOA comparison, suggesting a commonly overexpressed pathway shared between $\mathrm{IOA}$ and IA, with the genes mapping to a proliferative signature. The proliferation marker Ki67-encoding gene MKI67 was highly overexpressed in iOA compared with cOA $\left(7.2 \log _{2}\right.$ fold change; adjusted $P=0.013$ ). Between cOA vs. IA and cOA vs. iOA, only 9 upregulated genes intersect against 103 exclusive genes, signifying a difference in regulation of overexpressed genes. This is visualized in an UpSet plot ordered by frequency (Figure 4A).

To assess the link between increased numbers of macrophages and cell cycle gene signatures in iOA, we measured the protein expression of the proliferation marker Ki67 and assessed the frequency 
A
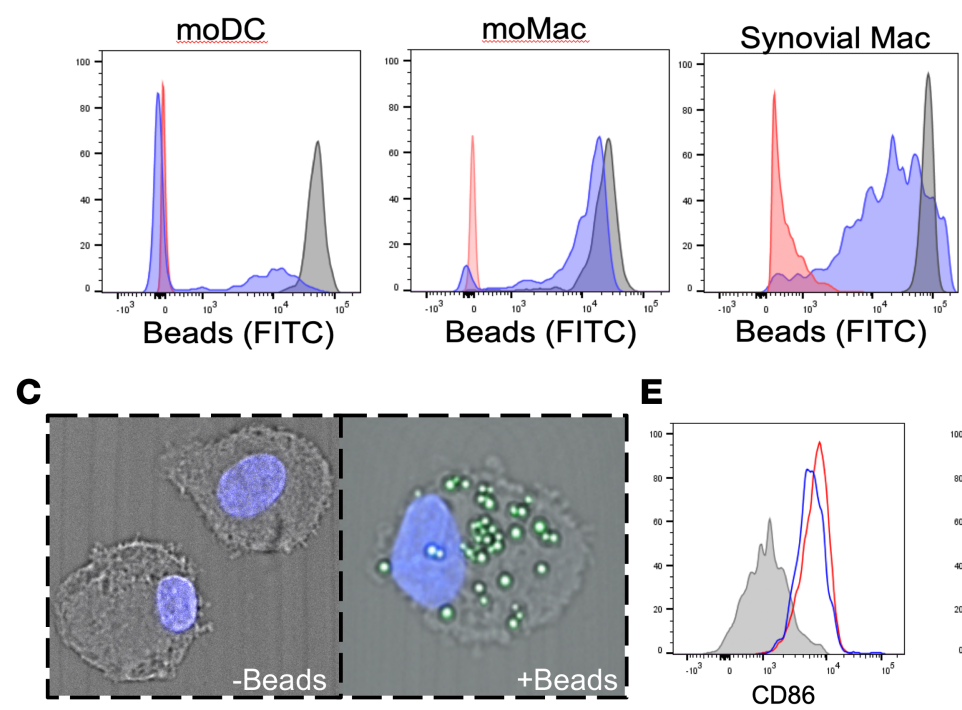

E
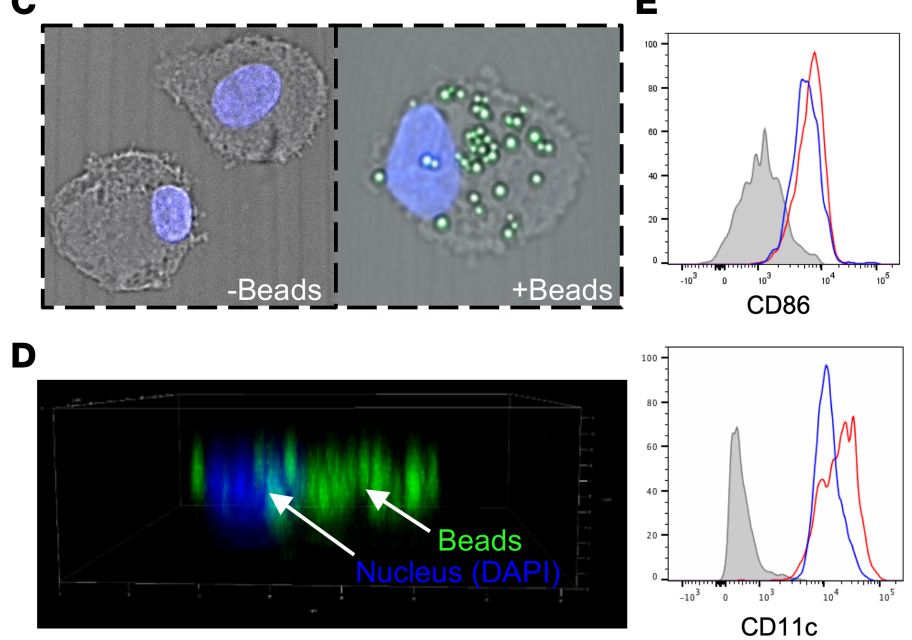
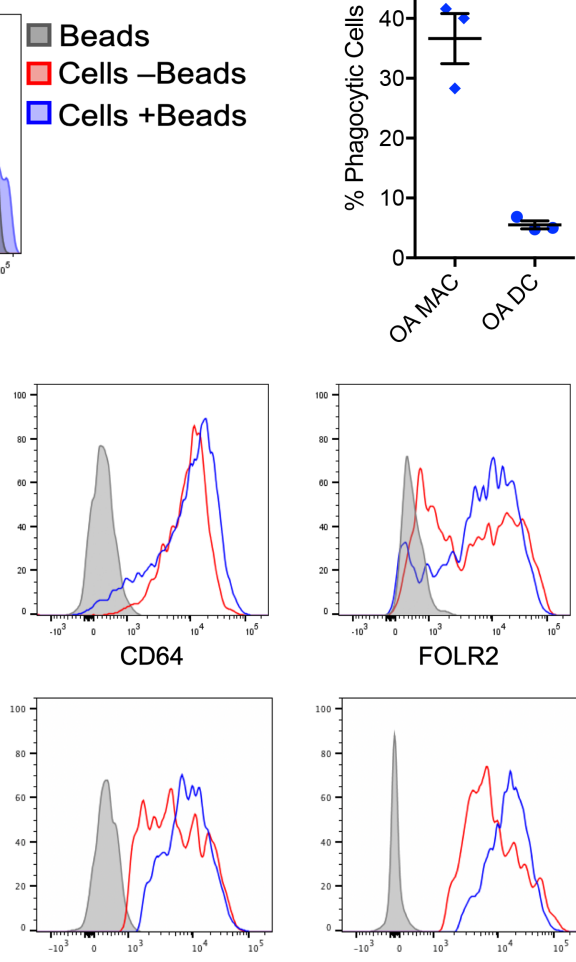

HLA-DR
B
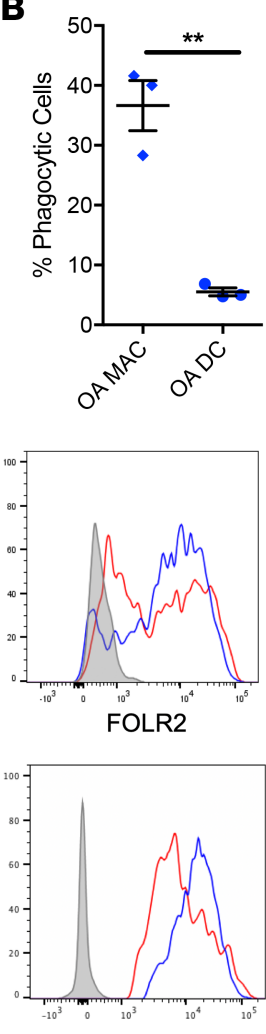

CD14

Figure 2. Synovial tissue macrophages. Synovial tissue from OA total knee replacement and IA ultrasound-guided biopsy was digested using optimized protocol. (A) Expression of latex bead fluorescence in FITC channel. Histograms depict OA synovial tissue macrophages incubated with beads (blue), cells incubated without beads (red), and beads alone (gray). Left panel depicts monocyte-derived DCs, middle panel depicts monocyte-derived macrophages, and right panel depicts OA synovial tissue macrophages. Data are representative of 3 independent experiments. mo, monocyte-derived. (B) Phagocytosis of latex beads by OA synovial tissue macrophages and DCs. $n=3$. (C) Confocal microscope image utilizing differential interference contrast (DIC). Left panel depicts OA synovial tissue macrophages cultured without latex beads. Right panel depicts OA synovial tissue macrophages cultured with latex beads. Images are representative of 3 individual experiments. Original magnification, 100x. (D) Confocal Z-stack reconstruction of 39 images of an $0 A$ synovial tissue macrophage. Blue areas indicate DAPI staining of nucleus. Green areas indicate latex beads. (E) Cell surface staining of CD86, CD64, and F0LR2 on synovial macrophages from OA (blue) and IA (red). FMO, gray. Data are representative of 4 experiments. ${ }^{* *} P \leq 0.01$ by 2 -tailed unpaired $t$ test.

of macrophages in OA subgroups by flow cytometry (Figure 4, C-E). This demonstrated that those synovial tissue samples with large quantities of macrophages ( $>30 \%$ of $\mathrm{CD} 45^{+}$cells; Figure $4 \mathrm{C}$ ) have a significantly higher proportion of macrophages that stain positive for Ki67 (Figure 4, D and E), perhaps suggesting that the increased proportion of synovial macrophages measured are directly contributed to by macrophage proliferation. Taken together, these data demonstrate that OA synovial macrophages have heterogeneous gene expression signatures that may represent differing cellular disease mechanisms and/or contrasting disease tissue environments.

\section{Discussion}

Through the in-depth characterization of synovial macrophages, we have been able to identify 2 distinct OA subgroups, cOA and iOA, which contain distinct macrophage populations with different functions. cOA macrophages were characterized by a tissue remodeling signature and were distinct from IA synovial macrophages. In contrast, $\mathrm{iOA}$ macrophages displayed a strong proliferation signature and were more closely aligned with macrophages from IA synovial tissue. This was further validated by flow cytometry, which distinguished the same iOA and cOA subgroups by macrophage frequency, indicating that the different macrophage gene signatures are associated with a differential macrophage abundance and proliferative characteristics. The expression of proliferation marker Ki67 was significantly higher in synovial tissues that contained larger numbers of macrophages, suggesting that the relative and absolute increase in macrophages in the iOA group is, at least in part, driven by cell division. 
A

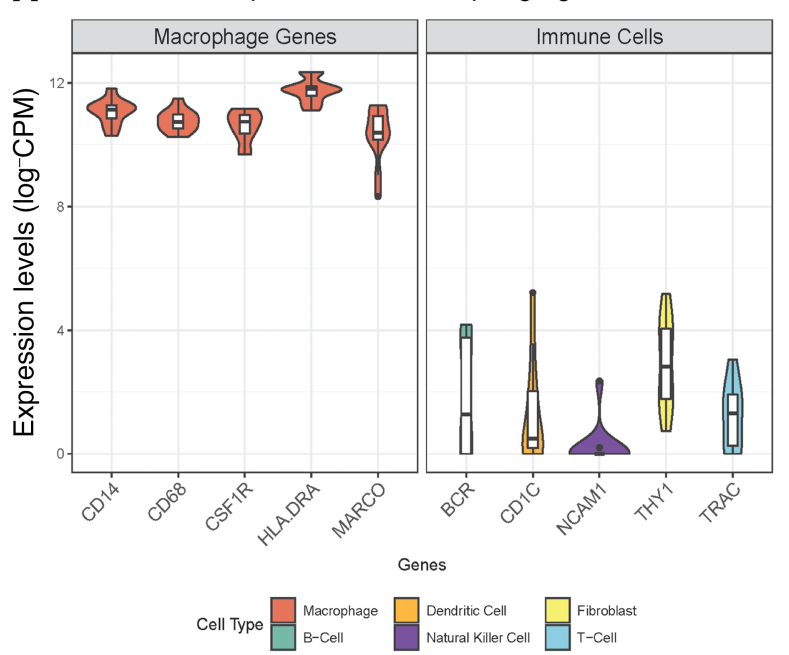

C

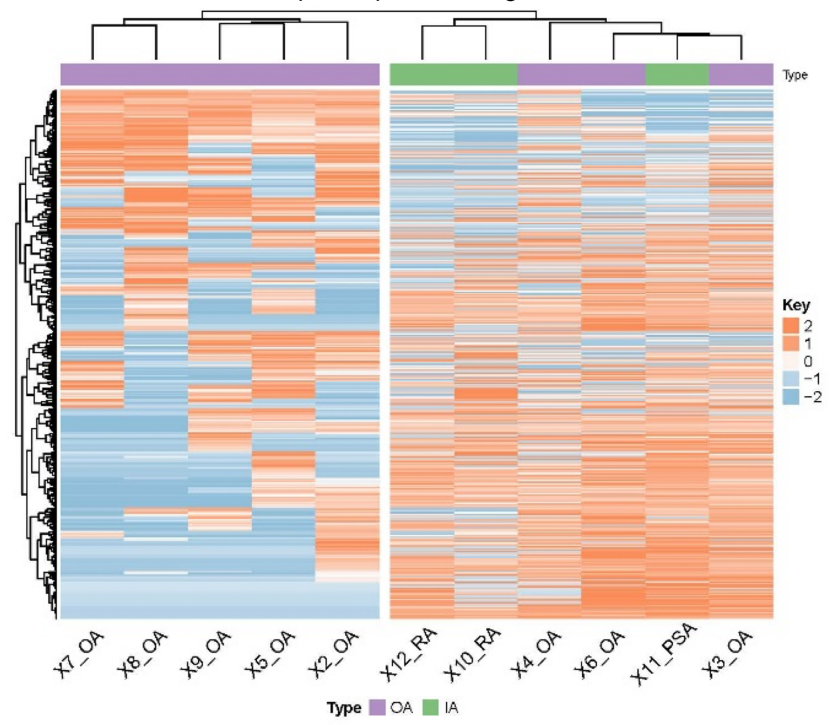

B

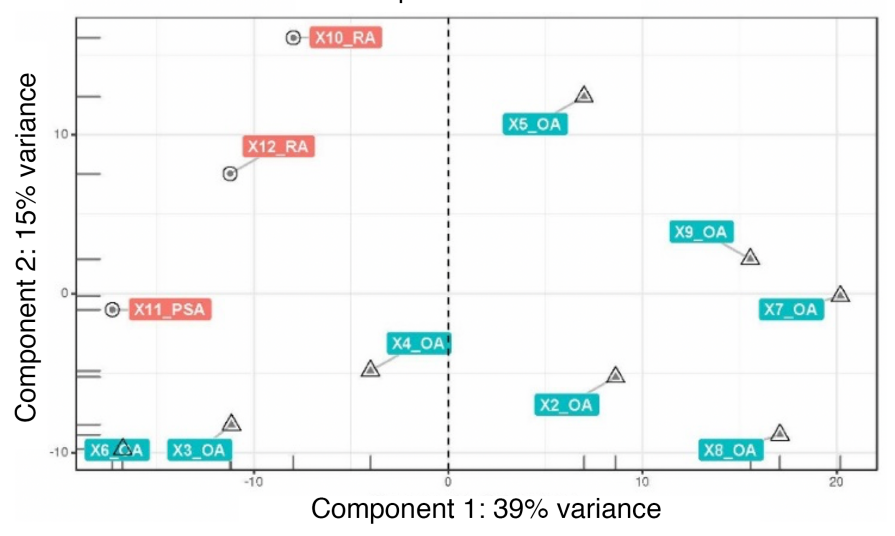

D Expression volcano across osteoarthritis groups

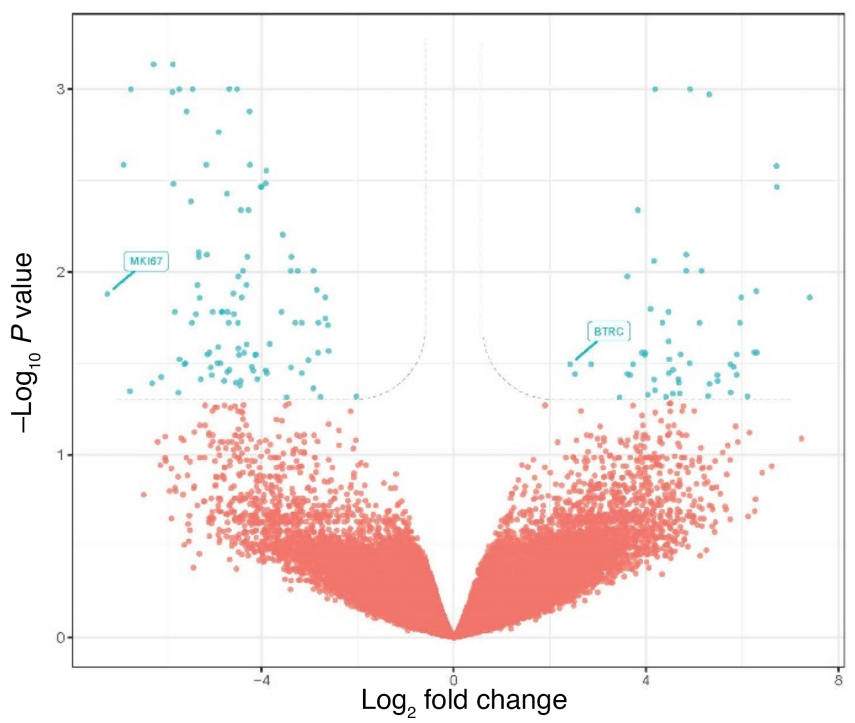

Figure 3. Identification of osteoarthritis synovial tissue macrophage heterogeneity. RNA sequencing of synovial macrophages and data analyses were conducted following description in methods. (A) Log counts per million (logCPM) expression levels of macrophage genes (left panel) and B cell (BCR), DC (CD1C), NK cell (NCAM1), stromal (THY1), and T cell (TRAC) genes (right panel). (B) PCA plot of all samples. Red text and circle identifies IA samples; blue text and triangle identifies OA samples. (C) Hierarchical clustering heatmap of top 500 genes across OA samples. (D) Volcano plot identifying significantly differentially expressed genes between COA and iOA ( $>1.5$-fold change; $P<0.05$, Benjamini-Hochberg multiple test corrected), identified by blue data points. Red data points identify nondifferentially expressed genes.

Macrophage proliferation and accumulation is usually seen under inflammatory conditions - for example, in response to infection with parasites (38) or in atherosclerotic plaques (39). Mouse model studies have furthermore shown that these proliferating macrophages are not derived from monocytes entering the inflamed tissue, but instead represent self-renewal of resident macrophages $(38,39)$. Our finding that synovial macrophages have a proliferation signature in a subset of OA patients is the first demonstration to our knowledge that a similar process may be going on in the OA synovium. Since proliferation of mouse tissue resident macrophages was driven by inflammation, our finding also strongly suggests that the proliferating macrophages signature identifies OA patients in whom synovial inflammation is part of disease pathogenesis.

Historically, macrophages have been described as either proinflammatory (M1) or antiinflammatory (M2), defined on the basis of monocyte responses to stimuli in vitro $(40,41)$. Our data indicate that synovial macrophages from OA or IA patients do not clearly align with this M1/M2 paradigm. Our findings are in line, however, with other studies investigating the function of tissue macrophages (42). Indeed, it is now 
A

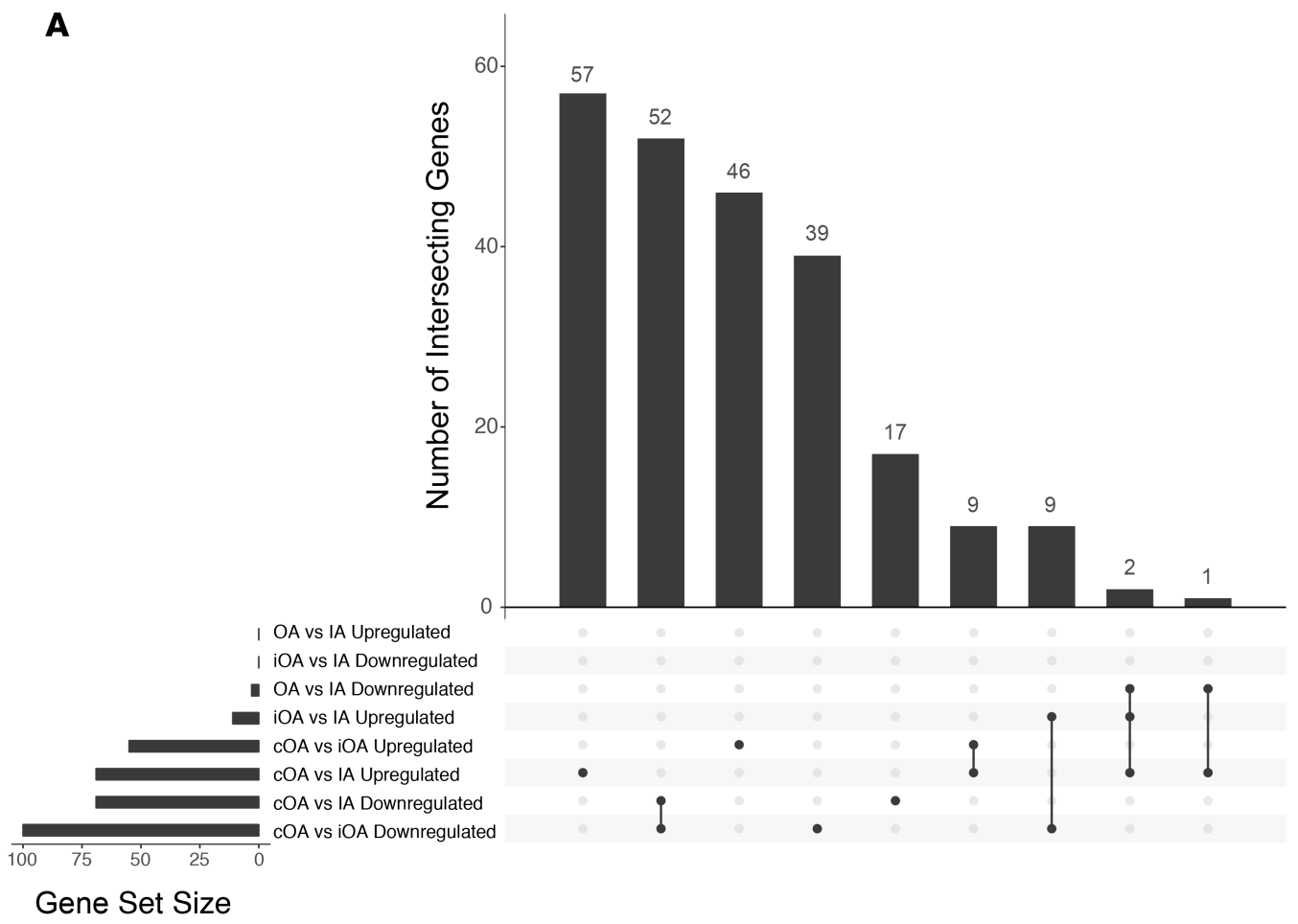

\section{B}

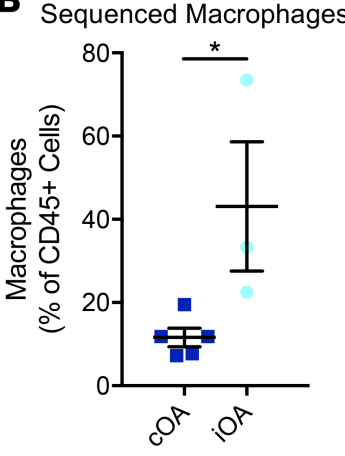

C

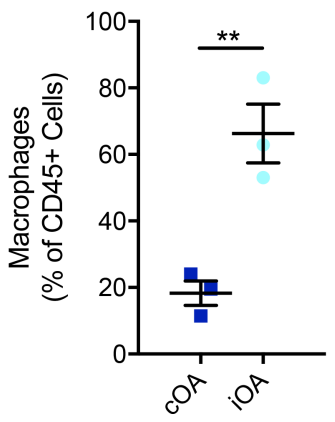

D

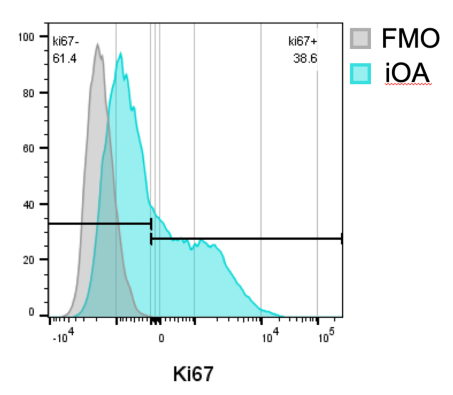

$\mathbf{E}$

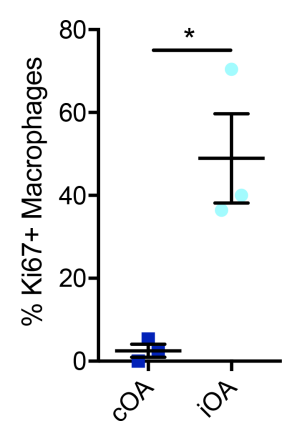

Figure 4. Functional gene set analysis of IOA and COA. RNA sequencing of synovial macrophages and data analyses were conducted following description in methods. (A) UpSet plot visualizing the sets of differentially expressed genes from different contrasts, including the quantitative analysis of aggregate intersections between comparisons. Sets ordered by frequency. DEGs ( $>1.5$-fold change; $P<0.05$, multiple test corrected) identified in $0 A$ vs. IA, iOA vs. IA, and $\mathrm{COA}$ vs. iOA. (B) Percentage of macrophages in $\mathrm{CD} 45^{+}$cell fraction of synovial tissue of RNA-sequenced samples. $n=8$. (C) Cell subsets measured as a percentage of $\mathrm{CD} 45^{+}$cell fraction isolated from synovial tissue. COA and iOA subgroups from additional samples identified initially by enumeration of synovial macrophages. $C O A \leq 30 \%$ macrophages; iOA $\geq 30 \%$ macrophages. $n=6$. (D) $\mathrm{KI} 67^{+}$macrophages in iOA group identified in $\mathbf{C}$. Data are representative of 6 individual experiments. (E) Enumeration of Ki67+ macrophages in $\mathrm{COA}$ and $\mathrm{OOA}$ groups identified in $\mathbf{C} . n=6 .{ }^{*} P \leq 0.05,{ }^{*} P \leq 0.01$ by 2-tailed unpaired $t$ test.

becoming accepted that M1 and M2 macrophages do not adequately reflect tissue macrophages in vivo; they are now regarded as polar extremes of a spectrum of in vitro macrophage activation states (43). Our RNA-Seq data shows that a mixture of both M1- and M2-related genes can be expressed by synovial macrophages across all patients. This could either reflect the presence of a mixture of M1- and M2-like polarized macrophages in the arthritic synovial tissues or differential activation states of these synovial macrophages within the 2 extremes of the M1/M2 spectrum. Either way, the M1/M2 activation state of the synovial macrophages does not appear to model the activation status of ex vivo synovial macrophages in disease.

The transcriptomic analyses revealed a substantial heterogeneity within the OA synovial macrophage samples. This heterogeneity, and lack of OA stratification, masked differences when performing differential gene expression analysis between IA and OA macrophages ( 3 genes were differentially expressed between the IA and OA subgroups [ $>1.5$ fold change; $P<0.05$, Benjamini-Hochberg multiple test corrected]; Supplemental Figure 5 and Supplemental Table 3). There has only been 1 previous study performing gene expression 
analysis on purified synovial macrophages from rheumatoid arthritis (RA) and OA patients (44). This recent paper also struggled to identify clear distinctions between RA and OA macrophage gene signatures and did not stratify OA patients. This failure to observe differences between RA and OA gene expression may be due to differences in flow cytometry/FACS sorting gating strategy; for example, if autofluorescence is not included, DCs and monocytes may also be captured with macrophages (data not shown), thus contributing to heterogeneous gene expression profiles of RNA-sequenced samples (44). These findings highlight that the classification of OA as a single disease group may not be appropriate for this heterogeneous disease, as well as the importance of using autofluorescence when identifying synovial macrophages.

Upon reclassifying OA samples into cOA and iOA, more differences were observed in differential gene expression between IA and cOA, as well as the 2 OA subgroups themselves, cOA vs. iOA. The observation of similar clustering in a parallel dataset (i.e., flow cytometry enumeration of immune cell subsets) supports our classification. Distinct functional gene signatures were further identified in cOA and iOA. cOA was defined by cartilage remodeling features, whereas iOA was characterized by a proliferation signature. Since IGFBP5 is associated with the negative regulation of inflammatory mediators, overexpression of this gene in cOA macrophages could indicate a less inflammatory tissue environment compared with IA. Likewise, overexpression of HTRA1 is observed in COA macrophages compared with IA. HTRA1 can modulate synovial fibroblasts to produce cartilage catabolic MMPs. Alongside the observed overexpression of EFEMP1, potentially acting as a negative regulator of chondrogenesis, this could demonstrate a potential mechanism of cartilage destruction and subsequent failure of cartilage repair in these cOA patients. SMAD3 was also observed to be overexpressed, and RUNX2 was observed to be underexpressed in cOA macrophages compared with both IA and iOA macrophages, again suggesting an additional failing tissue repair mechanism. The overexpression of $U L K 1$ in IA and iOA macrophages compared with cOA macrophages was also of interest. It was noted that expression of $U L K 1$ was absent in COA macrophages. $U L K 1$ is protective of cartilage and could present another mechanism of cartilage destruction through reduced autophagy and increased chondrocyte death (45).

iOA macrophages were more closely aligned to IA macrophages, and genes expressed had a strong association with cell cycle processes. The overexpression of MKI67 in iOA macrophages compared with cOA macrophages was of particular interest, since it encodes a well-associated protein of cell proliferation, Ki67. Indeed, iOA synovial tissue contained higher proportions of macrophages, and synovium samples with high proportions of macrophages expressed high levels of the protein Ki67. Additionally, E2F8 and CDT1, which also modulate cell proliferation thorough G1/S phase, were also overexpressed in iOA. As iOA macrophages were comparable in their gene expression profile to IA macrophages, this macrophage proliferation could be responding to an immune cell-driven inflammatory stromal interaction within the joint, as documented in IA (46). Overexpression of CTRL in iOA macrophages compared with cOA macrophages may also reflect a more inflammatory environment and result in further contribution to this inflammatory environment, creating a positive feedback loop.

Interestingly, the $\mathrm{iOA}$ and cOA subgroups did not correlate with any of the clinical data, including radiographic measures of disease severity (Kellgren-Lawrence X-ray score), indicators of systemic inflammation (C-reactive protein [CRP] and erythrocyte sedimentation rate $[E S R])$, or medication. This suggests that the distinction of 2 OA groups based on their macrophage gene signature cannot be predicted and is most likely not influenced by these parameters. Although the small sample size may have precluded any correlations between clinical data and the $\mathrm{iOA}$ and cOA groups (RNA-Seq cohort, $n=8$ ), correlations were also not found when analyzing the proportion of synovial macrophages (flow cytometry cohort, $n=64$ ) and the clinical parameters. Thus, for future stratification of OA patients for treatments targeting synovial inflammation, more specific biomarkers will need to be developed to indicate which subgroup OA patients belong to.

Taken together, these data suggest that there are divergent synovial tissue environments between cOA and iOA patients. It is likely that these environments specifically modulate synovial macrophages to differing patterns of gene expression. iOA appears to be particularly inflammatory, whereas cOA pathogenesis is likely to be centered around cartilage degradation. Within cOA macrophages, there are aberrant tissue repair mechanisms, in addition to factors that may directly contribute to cartilage degradation. iOA macrophages are highly proliferative, as reflected by their gene signature, their increased expression of Ki67, and their enhanced proportions within the synovium, as measured by flow cytometry. It is likely that the synovial environment directly contributes to these macrophages' proliferative capacity, but there is scope for a positive feedback loop whereby the macrophages further exacerbate synovial inflammation. 
The identification of the iOA and cOA subgroups confirms the long-postulated heterogeneity between OA patients. Recognizing and understanding these OA subgroups will allow us to explore and understand the heterogeneity of this disease. Ultimately, the stratification of OA patients for suitable disease-modifying treatments could lead to a true personalized medicine approach, in addition to the identification of novel therapeutic targets and biomarkers that can improve disease diagnosis and monitoring.

\section{Methods}

Cell isolation. Peripheral blood mononuclear cells (PBMCs) were isolated using density gradient centrifugation over Lymphoprep (Axis Shield). CD14 ${ }^{+}$monocytes were isolated using CD14 magnetic microbeads (Miltenyi Biotec) according to the manufacturer's protocol. Synovial tissue was dissociated into 1-mm fragments. Liberase TM (Roche Diagnostics) was added at a final concentration of $0.15 \mu \mathrm{g} / \mathrm{ml}$ and DNase (Roche Diagnostics) at $0.30 \mu \mathrm{g} / \mathrm{ml}$ and incubated in a shaking incubator $(260 \mathrm{rpm})$ at $37^{\circ} \mathrm{C}$ for 45 minutes, with additional vigorous shaking every 15 minutes. Solution was filtered with a $100-\mu \mathrm{m}$ filter, and filtrate was immediately placed on ice to stop enzymatic digestion. Any remaining tissue was subjected to repeated digestion steps.

Generation of monocyte-derived DCs and macrophages. CD14+ monocytes were cultured at $0.5 \times 10^{6} \mathrm{cells} / \mathrm{ml}$ in 24-well plates with $50 \mathrm{ng} / \mathrm{ml} \mathrm{IL-4} \mathrm{(Immunotools)} \mathrm{and} 50 \mathrm{ng} / \mathrm{ml}$ GM-CSF (Immunotools) for generation of DCs, or $50 \mathrm{ng} / \mathrm{ml} \mathrm{MCSF}$ (PeproTech) for generation of macrophages. On day 3, cytokine supplemented medium was refreshed. On day 6, cells were harvested, washed, and used in functional assays. Cells were cultured in RPMI 1640 (MilliporeSigma), supplemented with 10\% FBS (Thermo Fisher Scientific) and 2 $\mathrm{mM}$ glutamine, $100 \mathrm{U} / \mathrm{ml}$ penicillin, and $100 \mu \mathrm{g} / \mathrm{ml}$ streptomycin (all MilliporeSigma) at $37^{\circ} \mathrm{C}$ with $5 \% \mathrm{CO}_{2}$.

Flow cytometry staining, cell sorting, and analysis. For cell surface staining, single cell suspensions were incubated in FACS buffer (PBS + 3\% FBS (Life Technologies BRL) + 0.2\% EDTA (Fisher Scientific) + $0.1 \%$ sodium azide (Sigma-Aldrich)) + flow cytometry antibodies (Supplemental Table 7) + human IgG + viability dye. Flow cytometry and FACS sorting was performed on a BD Fortessa X20 or BD Aria FACS Fusion instrument, and data were analyzed using FlowJo10 (Tree Star Inc.). Flow cytometry gating strategy was based on fluorescence minus one (FMO), unstained, and isotype controls. For downstream applications involving cell culture, cells were sorted into cold culture medium. For genomic analysis, cells were sorted directly into RLT buffer (Qiagen) +1\% $\beta$-Mercaptoethanol (MilliporeSigma), vortexed, and immediately frozen on dry ice. For intracellular staining, cells were fixed and permeabilized using eBioscience intracellular fixation and permeabilization buffer set (eBioscience Intracellular Fixation \& Permeabilization Buffer Set), according to manufacturer's instructions. Cells were stained with Ki-67 antibody (Supplemental Table 7). Cells were preincubated with $2 \%$ mouse and rat serum (both MilliporeSigma) prior to antibody labeling.

Phagocytosis assay and confocal microscopy imaging. Fluorescent latex beads ( $1 \mu \mathrm{m}$; MilliporeSigma, L4655) were added to cells at a $1 / 4000$ dilution. Cells were incubated for 3 hours at $37^{\circ} \mathrm{C}$. After incubation, cells were harvested and washed 3 times with PBS to ensure removal of unbound beads or beads on the cell surface. Viability dye was added and bead uptake measured by flow cytometry. Slide preparations for confocal microscopy were carried out using CellTAK and mounted with Vectashield containing DAPI. Fluorescent latex beads (fluorescence excitation wavelengths $[\lambda \mathrm{ex}], \sim 470 \mathrm{~nm}$; emission wavelength [ $\lambda \mathrm{em}]$, $\sim 505 \mathrm{~nm}$ ) were imaged using Leica TCS SP8 confocal microscope, LAS X Software, and an EVOS FL Cell Imaging System (Invitrogen).

Cytospin and immunostaining. Cytospins were prepared by spinning FACS-purified cells onto cytospin slides at $600 \mathrm{~g}$ for 8 minutes using a cytocentrifuge (Shandon Cytospin 4; Thermo Fisher Scientific). Cytospin slides were fixed in methanol prior to Giemsa-Wright staining performed using Advia S60 (Siemens) with Hematek Wright-Giemsa stain PAK. Imaging was carried out using Olympus CKX41 inverted phase contrast microscope and Olympus CAMEDIA C-7070 digital camera.

$R N A$-Seq. RNA was isolated from FACS purified peripheral blood and synovial tissue cells using QIAGEN Micro RNeasy kit. Quantification and quality control was carried out using high-sensitivity RNA screen tape (Agilent Technologies) on an Agilent 2200 Tapestation. Samples were diluted accordingly to normalized quantities of RNA. Smart-seq v4 (Clontech) amplification was performed and Nextera XT DNA library prep kit was used. RNA-Seq was performed using Illumina NextSeq 500 at a 10 million, 75 bp read depth at the Genomics Core Facility (GCF) at Newcastle University led by Jonathan Coxhead. Quality control of RNA-Seq was carried out with FastQC. Read trimming was performed with Trimmomatic, mapping, and alignment with STAR and quantification with HTSEQ. SeqMONK was run to account for any DNA contamination. Counts were filtered to remove those of low number $(<0.5$ counts per million), 
followed by assessment of batch effect. All high-level analyses of RNA-Seq data were performed with R. After voom normalization of data, differential gene expression was performed using Limma, with an adjusted $P$ value threshold $<0.05$ based on Benjamini-Hochberg correction and log fold change $>1.5$. Exploratory Kyoto Encyclopaedia of Genes and Genomes (KEGG) pathway analysis and Gene Ontology (GO) GSEA were performed on differentially expressed genes after correcting for RNA length. For synovial tissue cellular characterization, 83 arthritis patients were collected (64 OA and 19 IA). For synovial tissue RNA-Seq analyses, 12 arthritis patients were collected (9 OA and 3 OA). Sample X1_OA was removed from further RNA-Seq analyses due to principal component analysis revealing that extrinsic influences unrelated to the biological conditions had skewed the data of this sample. To quantify the suspected outlier, we examined the eigenvalue variation along the first principal component, which determined this outlier to be beyond 3 SDs; this validates that this sample has likely been affected by processing or sequencing error and that the inclusion of this sample in further analyses would prove uninformative.

Data and materials availability. RNA-Seq data are publicly available (GEO accession no. GSE123492).

Statistics. Statistical analyses were carried out in Prism 5.03 (GraphPad Software), Minitab 17 Statistical Software (Minitab Inc.), and R version 3.4.3. Replicates for all data in main figures ranged from $n=3$ to $n=64$ and are specified in figure legends. Students $t$ tests performed were 2-tailed and unpaired. A $P$ value of less than 0.05 was considered significant. Data represent mean \pm SEM.

Study approval. This study was conducted according to Declaration of Helsinki principles. Healthy peripheral blood samples were obtained from volunteers with written informed consent under the Newcastle Autoimmune Inflammatory Rheumatic Diseases Research Biobank (NAIRD). Ethical approval was granted by the Newcastle and North Tyneside Research Ethics Committee (REC reference 12/NE/0395). OA arthritis synovial tissue from total knee replacement surgery was obtained with written informed consent through the Newcastle Bone and Joint Study (NBJS). Ethical approval was granted by the Newcastle and North Tyneside Research Ethics Committee (REC reference number 09/H0906/72). IA wrist synovial tissue was obtained with written informed consent by ultrasound-guided needle biopsy from patients with RA or psoriatic arthritis attending the Newcastle Early Arthritis Clinic (NEAC) who were themselves naive to immunomodulatory treatment, including steroids. Ethical approval was granted by the Newcastle and North Tyneside Research Ethics committee (REC reference number 12/NE/0251).

\section{Author contributions}

MJW, CMUH, SM, GR, MAH, and RS designed experiments; MJW and GR performed experiments; MJW and AL performed statistical and bioinformatic analyses; MJW, SM, MAH, JDI, and CMUH interpreted data; AGP and KSR supplied patient samples; MJW, SM, and CMUH drafted the manuscript; all authors were involved in discussing the data and provided feedback on the manuscript.

\section{Acknowledgments}

We acknowledge the Newcastle University Flow Cytometry Core Facility (FCCF) for assistance with the generation of flow cytometry data, the Newcastle University GCF for assistance with the generation of RNA-Seq data, and the Newcastle University Bioimaging Unit for assistance with the generation of confocal microscopy data. This work was funded by Versus Arthritis (formally Arthritis Research UK) (grant no. 20298) and the JGW Patterson (JGWP) Foundation. This project has received funding from the European Union's Horizon 2020 research and innovation program under the Marie Sklodowska-Curie grant agreement no. 654882. The research was supported by the NIH Research Newcastle Biomedical Research Centre based at Newcastle Hospitals NHS Foundation Trust and Newcastle University.

Address correspondence to: Catharien Hilkens, Institute of Cellular Medicine, Newcastle University, 3rd Floor William Leech Building, Faculty of Medical Sciences, Framlington Place, Newcastle upon Tyne, NE2 4HH, United Kingdom. Phone: 44.0.191.208.8026; Email: catharien.hilkens@newcastle.ac.uk.

MJW's present address is: Department of Microbiology and Immunology, University of Illinois at Chicago, Chicago, Illinois, USA.

AL's present address is: Division of Cancer and Genetics, Cardiff University, Cardiff, UK. 
1. Murray CJ, et al. The State of US Health, 1990-2010: burden of diseases, injuries and risk factors. JAMA. 2013;310(6):591-608.

2. Wallace IJ, et al. Knee osteoarthritis has doubled in prevalence since the mid-20th century. Proc Natl Acad Sci USA 2017;114(35):9332-9336.

3. Karsdal MA, et al. Disease-modifying treatments for osteoarthritis (DMOADs) of the knee and hip: lessons learned from failures and opportunities for the future. Osteoarthr Cartil. 2016;24(12):2013-2021.

4. Guerra ML, Singh PJ, Taylor NF. Early mobilization of patients who have had a hip or knee joint replacement reduces length of stay in hospital: a systematic review. Clin Rehabil. 2015;29(9):844-854.

5. National joint registry for England, Wales, Northern Ireland, and the Isle of Man. 13th Annual Report; 2016. http://www.njrreports. org.uk/Portals/0/PDFdownloads/NJR\%2013th\%20Annual\%20Report\%202016.pdf. Accessed December 20, 2018.

6. Bourne RB, Chesworth BM, Davis AM, Mahomed NN, Charron KD. Patient satisfaction after total knee arthroplasty: who is satisfied and who is not? Clin Orthop Relat Res. 2010;468(1):57-63.

7. Gunaratne R, Pratt DN, Banda J, Fick DP, Khan RJK, Robertson BW. Patient Dissatisfaction Following Total Knee Arthroplasty: A Systematic Review of the Literature. J Arthroplasty. 2017;32(12):3854-3860.

8. Sokolove J, Lepus CM. Role of inflammation in the pathogenesis of osteoarthritis: latest findings and interpretations. Ther $A d v$ Musculoskelet Dis. 2013;5(2):77-94.

9. Ayral X, Pickering EH, Woodworth TG, Mackillop N, Dougados M. Synovitis: a potential predictive factor of structural progression of medial tibiofemoral knee osteoarthritis -- results of a 1 year longitudinal arthroscopic study in 422 patients. Osteoarthr Cartil. 2005;13(5):361-367.

10. Torres L, et al. The relationship between specific tissue lesions and pain severity in persons with knee osteoarthritis. Osteoarthr Cartil. 2006;14(10):1033-1040.

11. Hill CL, et al. Synovitis detected on magnetic resonance imaging and its relation to pain and cartilage loss in knee osteoarthritis Ann Rheum Dis. 2007;66(12):1599-1603.

12. Baker K, et al. Relation of synovitis to knee pain using contrast-enhanced MRIs. Ann Rheum Dis. 2010;69(10):1779-1783.

13. Conaghan PG, et al. Clinical and ultrasonographic predictors of joint replacement for knee osteoarthritis: results from a large, 3-year, prospective EULAR study. Ann Rheum Dis. 2010;69(4):644-647.

14. Roemer FW, et al. Presence of MRI-detected joint effusion and synovitis increases the risk of cartilage loss in knees without osteoarthritis at 30-month follow-up: the MOST study. Ann Rheum Dis. 2011;70(10):1804-1809.

15. Scanzello CR, et al. Synovial inflammation in patients undergoing arthroscopic meniscectomy: molecular characterization and relationship to symptoms. Arthritis Rheum. 2011;63(2):391-400.

16. Soul J, et al. Stratification of knee osteoarthritis: two major patient subgroups identified by genome-wide expression analysis of articular cartilage. Ann Rheum Dis. 2018;77(3):423.

17. Driban JB, Sitler MR, Barbe MF, Balasubramanian E. Is osteoarthritis a heterogeneous disease that can be stratified into subsets? Clin Rheumatol. 2010;29(2):123-131

18. Kittelson AJ, George SZ, Maluf KS, Stevens-Lapsley JE. Future directions in painful knee osteoarthritis: harnessing complexity in a heterogeneous population. Phys Ther. 2014;94(3):422-432.

19. Kraus VB, Blanco FJ, Englund M, Karsdal MA, Lohmander LS. Call for standardized definitions of osteoarthritis and risk stratification for clinical trials and clinical use. Osteoarthr Cartil. 2015;23(8):1233-1241.

20. Tonge DP, Pearson MJ, Jones SW. The hallmarks of osteoarthritis and the potential to develop personalised disease-modifying pharmacological therapeutics. Osteoarthr Cartil. 2014;22(5):609-621.

21. Philp AM, Davis ET, Jones SW. Developing anti-inflammatory therapeutics for patients with osteoarthritis. Rheumatology (Oxford). 2017;56(6):869-881.

22. Hunter DJ. Are there promising biologic therapies for osteoarthritis? Curr Rheumatol Rep. 2008;10(1):19-25.

23. Smith MD. The normal synovium. Open Rheumatol J. 2011;5:100-106.

24. van Lent PL, et al. Crucial role of synovial lining macrophages in the promotion of transforming growth factor beta-mediated osteophyte formation. Arthritis Rheum. 2004;50(1):103-111.

25. Blom AB, et al. Synovial lining macrophages mediate osteophyte formation during experimental osteoarthritis. Osteoarthr Cartil. 2004;12(8):627-635

26. Bondeson J, Blom AB, Wainwright S, Hughes C, Caterson B, van den Berg WB. The role of synovial macrophages and macrophage-produced mediators in driving inflammatory and destructive responses in osteoarthritis. Arthritis Rheum. 2010;62(3):647-657.

27. Haywood L, et al. Inflammation and angiogenesis in osteoarthritis. Arthritis Rheum. 2003;48(8):2173-2177.

28. Benito MJ, Veale DJ, FitzGerald O, van den Berg WB, Bresnihan B. Synovial tissue inflammation in early and late osteoarthritis. Ann Rheum Dis. 2005;64(9):1263-1267.

29. Kraus VB, et al. Direct in vivo evidence of activated macrophages in human osteoarthritis. Osteoarthr Cartil. 2016;24(9):1613-1621.

30. Wu CL, et al. Conditional Macrophage Depletion Increases Inflammation and Does Not Inhibit the Development of Osteoarthritis in Obese Macrophage Fas-Induced Apoptosis-Transgenic Mice. Arthritis Rheumatol. 2017;69(9):1772-1783.

31. Gordon S. Alternative activation of macrophages. Nat Rev Immunol. 2003;3(1):23-35.

32. Blériot C, Dupuis T, Jouvion G, Eberl G, Disson O, Lecuit M. Liver-resident macrophage necroptosis orchestrates type 1 microbicidal inflammation and type-2-mediated tissue repair during bacterial infection. Immunity. 2015;42(1):145-158.

33. Amit I, Winter DR, Jung S. The role of the local environment and epigenetics in shaping macrophage identity and their effect on tissue homeostasis. Nat Immunol. 2016;17(1):18-25.

34. McGovern N, et al. Human dermal CD14 + cells are a transient population of monocyte-derived macrophages. Immunity. 2014;41(3):465-477.

35. Njoroge JM, Mitchell LB, Centola M, Kastner D, Raffeld M, Miller JL. Characterization of viable autofluorescent macrophages among cultured peripheral blood mononuclear cells. Cytometry. 2001;44(1):38-44.

36. Raine EV, Reynard LN, van de Laar IM, Bertoli-Avella AM, Loughlin J. Identification and analysis of a SMAD3 cis-acting eQTL operating in primary osteoarthritis and in the aneurysms and osteoarthritis syndrome. Osteoarthr Cartil. 2014;22(5):698-705.

37. Valdes AM, et al. Genetic variation in the SMAD3 gene is associated with hip and knee osteoarthritis. Arthritis Rheum. 2010;62(8):2347-2352. 
38. Jenkins SJ, et al. Local macrophage proliferation, rather than recruitment from the blood, is a signature of TH2 inflammation. Science. 2011;332(6035):1284-1288.

39. Robbins CS, et al. Local proliferation dominates lesional macrophage accumulation in atherosclerosis. Nat Med. 2013;19(9):1166-1172.

40. Mantovani A, Sica A, Sozzani S, Allavena P, Vecchi A, Locati M. The chemokine system in diverse forms of macrophage activation and polarization. Trends Immunol. 2004;25(12):677-686.

41. Krausgruber T, et al. IRF5 promotes inflammatory macrophage polarization and TH1-TH17 responses. Nat Immunol. 2011;12(3):231-238

42. Manferdini C, et al. From osteoarthritic synovium to synovial-derived cells characterization: synovial macrophages are key effector cells. Arthritis Res Ther. 2016;18:83.

43. Xue J, et al. Transcriptome-based network analysis reveals a spectrum model of human macrophage activation. Immunity. 2014;40(2):274-288

44. Mandelin AM 2nd, et al. Transcriptional Profiling of Synovial Macrophages Using Minimally Invasive Ultrasound-Guided Synovial Biopsies in Rheumatoid Arthritis. Arthritis Rheumatol. 2018;70(6):841-854.

45. Caramés B, Taniguchi N, Otsuki S, Blanco FJ, Lotz M. Autophagy is a protective mechanism in normal cartilage, and its aging-related loss is linked with cell death and osteoarthritis. Arthritis Rheum. 2010;62(3):791-801.

46. Juarez M, et al. DKK1 expression by synovial fibroblasts in very early rheumatoid arthritis associates with lymphocyte adhesion in an in vitro flow co-culture system. Arthritis Res Ther. 2016;18:14. 